\title{
Radioactive Source
}

National Cancer Institute

\section{Source}

National Cancer Institute. Radioactive Source. NCI Thesaurus. Code C94963.

The substance which is radioactive. 